\title{
Los paradigmas de la investigación: un acercamiento teórico para reflexionar desde el campo de la investigación educativa
}

Research Paradigms: A Theoretical Approach to Reflect from the Field of Educational Research

Os paradigmas de pesquisa: uma abordagem teórica para refletir a partir do campo da pesquisa educacional

Sorangela Miranda Beltrán

Universidad Externado de Colombia, Facultad de Ciencias de la Educación, Colombia sorangela.miranda@uexternado.edu.co https://orcid.org/0000-0002-0886-2647

Jorge Alexander Ortiz Bernal

Universidad Externado de Colombia, Facultad de Ciencias de la Educación, Colombia jorge.ortiz@uexternado.edu.co https://orcid.org/0000-0003-3906-5269 


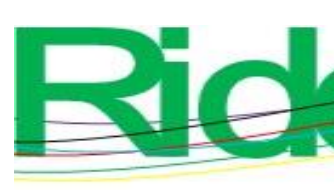

Revista Iberoamericana para la Investigación y el Desarrollo Educativo ISSN $2007-7467$

\section{Resumen}

El presente artículo académico busca responder la pregunta: ¿qué configuraciones teóricas se asumen desde los paradigmas de la investigación y qué nuevos retos se plantean en torno a ellos desde la investigación educativa? Para ello, se parte de la revisión teórica de los conceptos de conocimiento, epistemología y paradigma. Particularmente, se profundiza en el concepto de paradigma en cuanto que este sirve de marco para la comprensión de los fenómenos de la realidad y se identifican cuatro variantes: positivista (las teorías como verdades absolutas), interpretativo (la construcción de la realidad en las subjetividades), crítico (la emancipación para la transformación social) y el paradigma emergente de la complejidad. Una vez hecho el abordaje teórico, se determina que, en términos de la investigación educativa, es necesario generar reflexión frente a tres elementos relevantes: los retos investigativos frente a las dinámicas cambiantes de las sociedades, las miradas a los problemas propios de la educación y la postura del investigador en razón a los ámbitos del conocimiento desde los cuales parte para entender el fenómeno de estudio.

Palabras clave: complejidad, epistemología, investigación educativa, paradigma.

\section{Abstract}

This academic article seeks to answer the questions: What are the theoretical configurations assumed from the research paradigms and what are the new challenges posed around them from educational research? To do so, it starts with a theoretical review of the concepts of knowledge, epistemology and paradigm. The paradigm concept is deepened since it serves as a framework for the understanding of the phenomena of reality and the identification of four paradigms is revealed: positivist (theories as absolute truths), interpretative (the construction of reality in subjectivities), critical (the emancipation for social transformation) and the emerging paradigm of complexity. Once the theoretical approach has been made, it is determined that, in terms of educational research, it is necessary to generate reflection on three relevant elements: the research challenges in the face of the changing dynamics of societies, looks at the problems of education, and the position of the researcher in terms of the areas of knowledge from which they are supported in order to understand the phenomenon of study.

Keywords: complexity, epistemology, educational research, paradigm. 

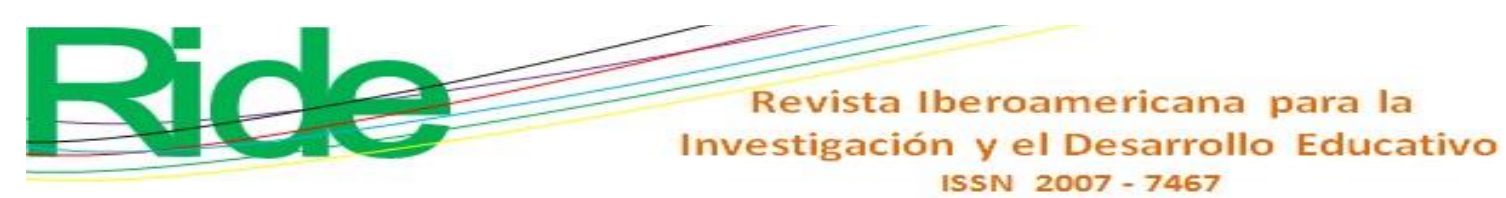

\section{Resumo}

Este artigo acadêmico busca responder à questão: quais configurações teóricas são assumidas a partir dos paradigmas de pesquisa e que novos desafios surgem em torno deles a partir da pesquisa educacional? Para tanto, partimos da revisão teórica dos conceitos de conhecimento, epistemologia e paradigma. Em particular, o conceito de paradigma é aprofundado na medida em que serve de arcabouço para a compreensão dos fenômenos da realidade e são identificadas quatro variantes: positivista (teorias como verdades absolutas), interpretativa (a construção da realidade nas subjetividades ), crítica (emancipação para a transformação social) e o paradigma emergente da complexidade. Feita a abordagem teórica, determina-se que, em termos de pesquisa educacional, é necessário gerar reflexão sobre três elementos relevantes: os desafios da pesquisa diante das dinâmicas mutantes das sociedades, as visões sobre os problemas da educação e a posição do pesquisador em relação às áreas do conhecimento a partir das quais passa a compreender o fenômeno de estudo.

Palavras-chave: complexidade, epistemologia, pesquisa educacional, paradigma.

Fecha Recepción: Febrero 2020

Fecha Aceptación: Agosto 2020

\section{Contextualización}

Plantear los aspectos que, desde el punto de vista teórico, permiten comprender el concepto de paradigma y su aplicación en el amplio campo de la investigación educativa es un ejercicio que implica volver la mirada al debate sobre el conocimiento como la posibilidad de acercarse al abordaje de las realidades y la epistemología como un punto de reflexión de las ciencias sobre aquello que se concibe como el proceso de conocer.

En efecto, la temática propuesta para este artículo exige sentar las bases para que, a partir de la teoría, se pueda lograr la comprensión sobre los valores, los saberes y los métodos que, desde lo científico, procuran responder a los problemas emergentes en las distintas dimensiones sociales y humanas. Para ello, la presente relatoría tiene como intención atender a la pregunta bipartita: ¿qué configuraciones teóricas se asumen desde los paradigmas de la investigación y qué nuevos retos se plantean en torno a ellos desde la investigación educativa? Se trata de interrogantes que se propone sean abordadas desde la revisión de los diferentes autores, las nociones conceptuales y las escuelas de pensamiento que han incidido de manera significativa en plantear posibles definiciones de las categorías de conocimiento, 


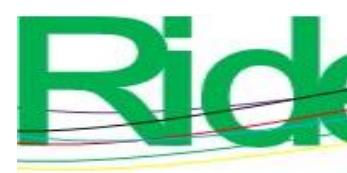

\section{Revista Iberoamericana para la Investigación y el Desarrollo Educativo ISSN 2007 - 7467}

Desde esta perspectiva, el conocimiento científico brinda un marco específico a la noción de paradigma, ya que establece presupuestos de orden metodológico, ontológico y epistémico que son avalados y aceptados por una comunidad de científicos.

\section{El Paradigma y sus tipologías: elementos de análisis}

En su obra La estructura de las revoluciones científicas, Kuhn (1962) define paradigma como "un conjunto de suposiciones interrelacionadas respecto al mundo social que proporciona un marco filosófico para el estudio organizado de este mundo" (p. 82). Esta perspectiva ubica al paradigma como una estructura de índole conceptual, fundamentada en creencias y supuestos teóricos y metodológicos, que, tal y como lo afirma el autor, orienta a una determinada comunidad científica dentro de su cosmovisión sobre el mundo, lo cual afianza los valores y saberes que comparten como colectivo (Marín, 2007).

La cosmovisión del mundo planteada por Kuhn (1962) es retomada por Patton (1978, citado en Medina, 2001). Siguiendo a este último teórico, un paradigma es "una perspectiva general, un modo de desmenuzar la complejidad del mundo real” (p. 81). Además, de acuerdo con esta misma fuente, los paradigmas subrayan los aspectos relevantes, legítimos y razonables. Sin duda estos planteamientos han asentado las bases para comprender cómo un paradigma influye en la concepción del mundo y la manera en que se abordan los problemas emergentes. De ahí que, según Marín (2007), el paradigma sea crucial desde un punto de vista aplicativo, a la hora de determinar las maneras de concebir el mundo, lo cual se refleja en la formulación de leyes, lenguajes y valoraciones. De este modo, para este pensador, el paradigma representa una acumulación de saber que se adhiere a la comprensión de ciertos fenómenos y permite resolver sus anomalías, y para las comunidades científicas representa tanto creencias como técnicas y valores que les permiten abordar los problemas y plantear sus soluciones.

Dentro de este marco conceptual, Morín (citado en Marín, 2007) reflexiona sobre los conceptos fundamentales o categorías rectoras y las relaciones lógicas de atracción y repulsión entre dichos conceptos y dichas categorías que imprimen sellos semánticos e ideológicos para darle sentido a las formas de ser, actuar y pensar de las comunidades que se adhieren a un paradigma en específico.

Lo anterior se reafirma en lo expresado por Alvarado y García (2008) cuando argumentan que los paradigmas se consolidan como patrones o modelos que recopilan las 


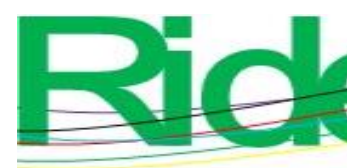

Revista Iberoamericana para la
Investigación y el Desarrollo Educativo
ISSN $2007-7467$

creencias, reglas, presupuestos y procedimientos sobre los cuales los investigadores se disponen a hacer ciencia. Al respecto, Lukas y Santiago (2009) subrayan que un paradigma no es un posicionamiento personal, sino algo compartido y legitimado por una comunidad científica.

Así pues, el paradigma se confirma como una estructura conceptual que, como lo respalda Najmanovich (19 de octubre de 1991), permite desarrollar la investigación en áreas determinadas a partir de entidades que las fundamentan y que, a través de diferentes técnicas, procuran buscar soluciones a determinados problemas. Asimismo, precisa Marín (2007), dichas entidades se basan en operaciones lógicas e ideológicas que brindan un marco comprensible y coherente para abordar la realidad y generar nuevos conocimientos. En suma, es posible afirmar que los paradigmas sirven de marco para la comprensión de los fenómenos de la realidad; brindan una guía para abordar cuestiones y problemáticas; otorgan, dentro de un esquema de criterios, las técnicas apropiadas y la epistemología coherente para abordar situaciones emergentes (Medina, 2001).

En otras palabras, el paradigma provee al investigador la teoría y los referentes metodológicos para acceder al fenómeno objeto de estudio y, como lo indica Vasilachis (2006), proporciona un sistema filosófico y de investigación para comprender las cuestiones propias del proceso investigativo.

Estas últimas afirmaciones ponen en evidencia cómo a partir del paradigma desde el cual se ubique el investigador se aplican distintos métodos, principios e instrumentos durante la labor de investigación, lo cual otorga cualidades y singularidades propias a cada uno de los acercamientos (Ricoy, 2006).

Finalmente, como aspectos claves para comprender su alcance en el escenario de la construcción de los conocimientos y en el abordaje investigativo en educación, es imprescindible mencionar las características claramente definidas con las que Ricoy (2006) y Marín (2007) revisten a un paradigma. A continuación, parafraseando a los mencionados autores, se enlistan estas características:

- $\quad$ Su imposibilidad de invalidación, falsación o destrucción, toda vez que el paradigma se somete a una pérdida de vigencia, mas no a alternativas donde sean clasificados como verdaderos o falsos. Sus planteamientos filosóficos, sus concepciones y las técnicas para abordar los problemas se van transformando en la medida en que se generan revoluciones que, a la manera de Kuhn (1962), implican nuevos retos para repensar la realidad desde la postura de las disciplinas científicas. 


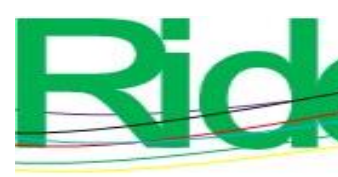

Revista Iberoamericana para la
Investigación y el Desarrollo Educativo
ISSN $2007-7467$

- Se reafirma a sí mismo en términos de su exclusividad, reconociendo únicamente sus propias creencias, métodos y valores, sin posibilidad de aceptar otras nociones.

Se consolida sobre un sistema filosófico y de investigación (Vasilachis, 2006) que brinda un orden en la comprensión científica, anteponiendo supuestos epistemológicos desde los cuales se conoce la realidad y se abordan los problemas de esta.

Derivado de estas conceptualizaciones sobre la categoría de paradigma, la pregunta que nos asiste en el marco de la presente reflexión es de cuántos paradigmas podríamos hablar. Se trata de una discusión sobre la cual la literatura especializada no ha logrado unanimidad: a tal grado que algunos expertos hacen referencia a dos paradigmas, otros hablan de tres, inclusive algunos más hablan del devenir del paradigma ecológico, también llamado visión holística del mundo. Teniendo en cuenta lo anterior, y con el propósito de continuar la discusión académica sobre el tema, a continuación presentamos cuatro paradigmas que están presentes en el escenario de la investigación educativa.

\section{El paradigma positivista. Las teorías como verdades absolutas}

Este paradigma se ubica dentro de la teoría positivista; plantea la posibilidad de llegar a verdades absolutas en la medida en que se abordan los problemas y se establece una distancia significativa entre el investigador y el objeto de estudio. Desde el punto de vista epistemológico, este paradigma brinda una distinción entre quien investiga como un sujeto neutral y la realidad abordada que se asume como ajena a las influencias del sujeto científico.

Dentro de esta concepción, Flores (2004) analiza cómo desde el positivismo se opta por una postura ontológica que posiciona a la realidad dentro del dominio de leyes naturales y mecanismos. "El conocimiento de estas leyes y mecanismos es convencionalmente resumido en la forma de tiempo y generalizaciones independientes del contexto. Algunas de estas generalizaciones toman la forma de leyes causa-efecto" (Flores, 2004, p. 4). A causa de ello, las ciencias físicas y naturales y, en las últimas décadas, las ciencias sociales y humanas han adoptado este paradigma.

Usher y Bryant (1992, citados en Ricoy, 2006) establecen unos supuestos básicos de esta óptica positivista que se sintetizan a continuación: 


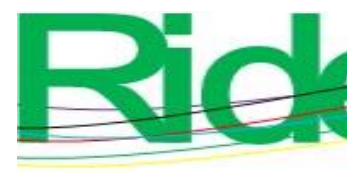

Revista Iberoamericana para la Investigación y el Desarrollo Educativo ISSN $2007-7467$

- La despersonalización de los individuos, al considerar la existencia de un mundo real fuera de ellos y un conocimiento de ese mundo a partir de métodos y procedimientos empíricos.

- La posibilidad de conocer la realidad mediante fenómenos observables, asumiendo el conocimiento como algo objetivo y susceptible de medición, considerando la producción de los saberes alejada de los compromisos de valor, aspecto que inspira la naturaleza cuantitativa del conocimiento en la medida en que se procura por el alcance de un saber comprobable y con posibilidad de ser comparado y replicable.

Adicional a estas características, se hace necesario reafirmar que, desde este paradigma, se parte de un sistema hipotético deductivo que reafirma la relevancia de hacer del conocimiento un proceso sistemático y medible dentro del marco del control empírico y el análisis causal de los fenómenos, elementos que, para la investigación en educación, implican la separación de las nociones subjetivas de quienes se hacen partícipes en el ejercicio investigativo para proveer principios y leyes que, derivadas de diseños metodológicos apoyados en las ciencias exactas, hagan plausible la comprensión de la realidad expresada en generalizaciones, explicaciones aplicables en el ámbito universal y análisis de variables dentro de la relaciones causa-efecto, dependencia-independencia. Desde este paradigma, pues, se desarrolla una perspectiva del conocimiento que subordina lo situacional a lo generalizable, y se establece un método hipotético-deductivo en el cual se hace una sujeción del objeto estudiado al diseño metodológico.

Al respecto, Martínez (2013) aporta a la reflexión la consideración de tres momentos para el desarrollo de este método dentro de la lógica positivista: el primero tiene que ver con la construcción del objeto de estudio, comprendiendo la formulación hipotética del problema abordado; el segundo, relacionado con el diseño metodológico de la investigación, apoyado en la elección de técnicas de recopilación de datos que provean las posibilidades de medición, comprobación y comparabilidad, incluyendo instrumentos para la estandarización y verificación de los datos, y el tercero, la discusión y presentación de resultados que dan cuenta del alcance del ejercicio de medición. 


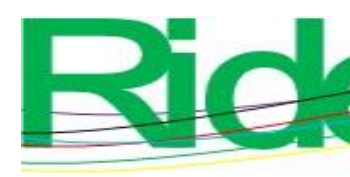

Revista Iberoamericana para la Investigación y el Desarrollo Educativo ISSN $2007-7467$

\section{El paradigma interpretativo. La construcción de la realidad en las subjetividades}

Como lo afirma Martínez (2013), el paradigma interpretativo surge como alternativa al paradigma positivista. Toma como punto de partida la idea de la dificultad para comprender la realidad social desde las lógicas cuantitativas, razón por la que este paradigma se fundamenta en las subjetividades y da cabida a la comprensión del mundo desde la apropiación que de él hacen los individuos.

Desde la interpretación como norte epistemológico, se promueve el análisis situacional del fenómeno. Una vez que se entienden sus particularidades, se posibilita el desarrollo de metodologías que procuran entender y significar las relaciones que se establecen en la singularidad de las realidades que confluyen en los distintos escenarios sociales, y así proveer múltiples datos, diversas perspectivas y formas de darle sentido al mundo de la vida (Habermas, 1985, citado en Hoyos, 1986). De ahí que, inherente a este paradigma, se ubiquen los enfoques cualitativos de la investigación desde miradas multimétodo, lo que le brinda al investigador la opción de valerse de información diversa para dar posibles respuestas a su pregunta de estudio.

Este paradigma encuentra su razón de ser en las dimensiones, en el sentido de que toma en cuenta las experiencias para el entendimiento del mundo y reconoce en la configuración de las subjetividades la influencia de aspectos históricos, culturales y sociales. Así, el conocimiento puede asumirse como el resultado de un ejercicio de construcción humana que no concluye al acercarse a las respuestas y soluciones frente a los problemas, sino que se transforma y abre a otras posibilidades epistemológicas.

La lógica interpretativa da cabida a dos corrientes filosóficas que fundamentan su método para acceder a los conocimientos: la hermenéutica y la fenomenología. La primera, según Martínez y Ríos (2006), plantea el acceso al conocimiento a través del estudio de las construcciones discursivas de un autor, una ciencia, una cultura, etc, con el propósito de comprender su significado (sentido). $\mathrm{Y}$ en ese sentido, la hermenéutica sostiene la no existencia de un saber objetivo, transparente, ni desinteresado sobre el mundo (Martínez y Ríos, 2006, p. 32).

La hermenéutica se apoya en la dialéctica, en la construcción del discurso para la comprensión y confrontación sobre los fenómenos del mundo, en el juicio reflexivo. Gadamer (1995) la propone como una forma de acceder a los conocimientos y 

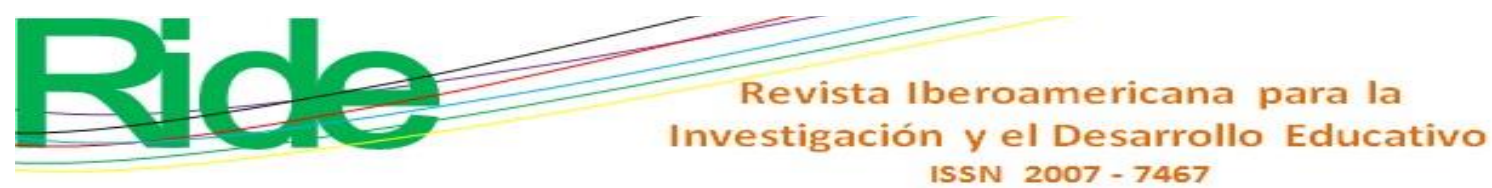

transformarlos. Aquí el reconocimiento del otro permite la construcción de los saberes y la captación del sentido que cada uno tiene de la realidad que interioriza y que apropia.

La fenomenología, por su parte, enfatiza en el ser y la conciencia; no descarta la experiencia sensorial que resulta de las vivencias y la interacción, de la participación en y con el objeto de estudio. El investigador, por tanto, adquiere una importante trascendencia en su ejercicio investigativo, toda vez que valida su participación en el proceso y enfatiza en sus intuiciones, sus sentidos, sus experiencias y conocimientos para interpretar esa realidad que percibe y que se alimenta de diferentes perspectivas.

Heidegger, Sartre y Husserl son sus más reconocidos representantes. La educación y las ciencias sociales y humanas se han servido de sus aportaciones para comprender cómo las experiencias contribuyen a configurar el mundo ideológico y pueden llegar a ser clave para acceder a los saberes y a los distintos tipos de conocimiento.

Entre las corrientes filosóficas mencionadas, Ricoy (2006) resalta las principales características del paradigma interpretativo en el ámbito de la investigación: especifica la relevancia que para este tiene profundizar en las tareas investigativas, rescata las posibilidades de generar diseños metodológicos surgidos de escenarios contextuales que atiendan a la participación de quienes hacen parte del proceso, reafirma el diálogo y la discusión productiva para generar conocimiento y comprender las diversas situaciones problémicas.

Estas consideraciones hacen posible vislumbrar el alcance ontológico de la lógica interpretativa, que, como hemos dicho, se apoya en la realidad asumida como producto de una construcción social, en la cual se admite la coexistencia de varias perspectivas y la pluralidad de visiones que se articulan en la comunicación y la interpretación dentro de los ambientes naturales de donde emergen los fenómenos.

\section{El paradigma crítico. La emancipación para la transformación social}

El paradigma crítico sienta su base en la teoría crítica del conocimiento que posiciona la reflexión y la emancipación social como respuesta a las hegemonías y las formas de dominio, y hace de la conciencia el medio para lograr las reivindicaciones frente a la justicia social y el alcance del bien común.

Este paradigma, que se inspira en los aportes de teóricos como Marcuse, Giroux, Habermas y Freire, plantea una mirada de la ciencia desde un punto de vista crítico y alude a la importancia de generar acciones que contribuyan a la transformación y emancipación del 


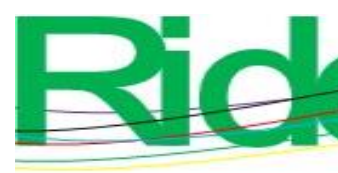

Revista Iberoamericana para la
Investigación y el Desarrollo Educativo
ISSN $2007-7467$

sujeto, con miras a romper las ataduras que le impiden reflexionar sobre su mundo y actuar de manera consciente en él.

Desde la postura crítica-reflexiva, los sujetos recrean sus realidades y, como lo expresa Ricoy (2006), pueden transformarlas de manera creativa al permitir la convergencia de criterios y al darle apertura a las visiones holísticas que le brindan sentido al mundo de la vida (Habermas, 1985, citado en Hoyos, 1986).

Los procesos investigativos son vistos como espacios de participación, responsabilidad social y compromiso frente a las necesidades y expectativas de las comunidades que, con base en las apuestas por la transformación emancipatorias, transitan por el camino para la toma de decisiones en beneficio común.

En este recorrido, las prácticas sociales se orientan con base en una teoría que se interioriza, reflexiona y analiza críticamente para ponerla en función de los cambios que se necesitan, valiéndose para ello de los actos comunicativos.

En este punto de la discusión, nuevamente los aportes de Habermas cobran relevancia al analizar cómo ese diálogo bidireccional, de colaboración y entendimiento, al que Freire (1980) alude cuando invita a poner en práctica una educación como práctica de libertad, se concreta en la comprensión de los significados que se logra a través de la participación. De acuerdo con Alvarado y García (2008):

Habermas parte del concepto de acción comunicativa porque él cree que es centralmente constitutivo de la sociedad humana; por ello trata, a partir de dicho concepto de reconstruir una filosofía de la racionalidad. Para este autor la razón es una trama discursiva que articula las acciones de los individuos (p. 5).

De esta manera, se reafirma que a través del discurso es posible encontrar formas de entendimiento que viabilicen el conocimiento de las realidades y orienten los saberes hacia una emancipación más concreta y hacia una toma decisiones con base en la autorreflexión y la conciencia crítica. En el ámbito educativo, por su parte, dichas nociones involucran asumir visiones globales y dialécticas de las realidades que confluyen en los contextos educativos, así como aceptar formas democráticas y participativas al momento de construir conocimiento. 


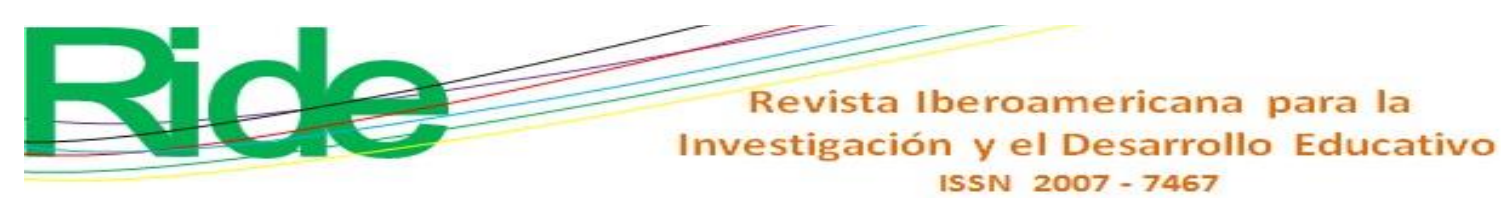

\section{El paradigma emergente de la complejidad}

Marín (2007) parte de la idea de que los sujetos construyen el conocimiento alrededor de incertidumbres y asumen la verdad como algo parcial que solo permite la inmersión en una parte del saber, lo cual desequilibra las posturas radicales en las que el conocer se enmarca en absolutismos, para convocar una lógica que incluye la indagación en un sentido abstracto, dialógico y complejo.

Coincidiendo con esta postura, Najmanovich (19 de octubre de 1991) plantea que el paradigma de la complejidad se basa en la teoría general de los sistemas, desarrollada inicialmente por Bogdanov y luego por Bertalanffy, la cual se apoya en un punto de vista transdisciplinario para avanzar en la comprensión de lo existente a partir del análisis del fenómeno en sus múltiples relaciones. Najmanovich (19 de octubre de 1991) añade que la atención se centra en "la estructura, la adaptación y el equilibrio dinámico, con el propósito de comprender cómo una organización dada podría ser estabilizada y mantenida por medio del cambio continuo" (p 2).

Lo expuesto se complementa con la preocupación por entender dichos cambios y los diferentes factores que inciden en estas transformaciones, y da paso a formas emergentes de asumir los conceptos y los diferentes componentes de la realidad.

Morín (citado en Najmanovich, 19 de octubre de 1991), como uno de los representantes destacados dentro de la teoría de la complejidad que encierra este paradigma, propone una visión integral y articulada de las dimensiones de bioantropología cosmológica y social, para abordar la comprensión de los cambios a partir de sus permanentes equilibrios y desequilibrios, dentro de una lógica de apertura a las posibilidades de transformación, y lo que implica esta lógica para quien asume el desafío de investigar en las condiciones de un mundo en constante caos.

El paradigma holístico o de la complejidad establece el conocimiento del mundo como una red inseparable de relaciones, ya que concibe al mundo como un todo integrado más que como una discontinua colección de partes, lo que, en términos generales, afecta al concepto tradicional de objetividad científica. En ese sentido, Capra (1998) indica que en el paradigma científico cartesiano las descripciones son consideradas objetivas, es decir, independientes del observador humano y del proceso de conocimiento. El nuevo paradigma, afirma este autor, trae consigo que la epistemología debe ser incluida explícitamente en la descripción de los fenómenos naturales. 


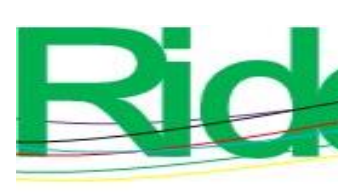

Revista Iberoamericana para la Investigación y el Desarrollo Educativo ISSN 2007-7467

\section{Apuntes finales}

Como se evidencia en el desarrollo del presente artículo, los aportes para la conceptualización de paradigma se consolidan como planteamientos fundamentales para entender la manera en que las comunidades científicas asumen modelos que les permiten pensar en los problemas y buscar soluciones frente a ellos. En términos de la investigación educativa, una vez más, implica convocar a la reflexión respecto a tres elementos relevantes: el primero, los retos investigativos en relación con las dinámicas cambiantes de las sociedades; el segundo, las miradas a los problemas propios de la educación, y el tercero, la postura del investigador en razón de los ámbitos del conocimiento desde los cuales se apoya para entender el fenómeno de estudio.

En cuanto al primer aspecto, es posible afirmar que las transformaciones permanentes de las sociedades exigen a los investigadores actitudes que les permitan asumir posiciones crítico-analíticas para comprender las complejidades y dinámicas del mundo, abordar las distintas realidades desde perspectivas que hagan posible la explicación de los fenómenos y plantear ideas que conduzcan a la articulación de las diversas teorías, conceptos, valores y nociones ontológicas para intentar responder científicamente a las preguntas que emergen, se profundizan y transforman en la interacción con los otros y con el entorno.

En este sentido, desde la investigación educativa, se hace un llamado a extender las posibilidades de indagación y búsqueda en las alternativas que dentro de los paradigmas se ofrecen. Esto para atender a los problemas sociales y humanos en el marco de procedimientos confiables, sistemáticos, flexibles y situados, significándolos en ámbitos de creencias y conocimientos que conlleven a discusiones académicas y amplios debates de cara a las realidades, en un ejercicio de apertura hacia nortes epistemológicos que ofrezcan una mayor comprensión y la posibilidad de una mayor intervención.

Los problemas de la humanidad, aquellos que se conocen y que aún no han podido ser superados, tal y como el hambre, la pobreza, la exclusión social, la desigualdad, los conflictos, la contaminación ambiental; aquellos emergentes producto de las relaciones culturales que se establecen en un mundo caracterizado por la diversidad, la multiculturalidad y la diferencia; aquellos que fluctúan en las transformaciones que la evolución de las ciencias

y las tecnologías traen para nuestro tiempo; aquellos que han puesto en crisis a la humanidad como la pandemia mundial generada por el covid-19; todos estos problemas, más otros que aún no conocemos y que devienen con los cambios acelerados de la sociedad, demandan 


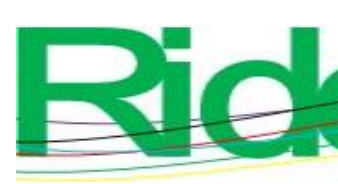

Revista Iberoamericana para la Investigación y el Desarrollo Educativo ISSN 2007 - 7467

profundas reflexiones, nuevas maneras de hacer investigación y otras apuestas para transformar los conocimientos dentro del quehacer investigativo cotidiano, así como desarrollar métodos y estrategias que permitan la identificación y resolución de los problemas y así ser capaces de responder a las vertiginosas transformaciones del mundo actual.

Así, el abordaje de las realidades en la apertura investigativa mencionada implica claridad para el investigador sobre el enfoque, la tradición y la perspectiva desde la cual desarrollará el tema de estudio (es decir, el paradigma en el que se ubicará para asumirlo conceptual y metodológicamente). Lo anterior supone, además, la adopción de un marco de saberes científicos firmemente consolidados y el establecimiento de unos criterios que, atendiendo a una rigurosidad metodológica que brinde confiabilidad y fiabilidad al ejercicio investigativo, retomen las dimensiones éticas, teóricas, contextuales, de responsabilidad social y ética que implica la intervención en el campo educativo.

En relación con el segundo aspecto, a saber, la mirada a los problemas propios de la educación, sigue siendo un reto comprender, profundizar y buscar alternativas ante las situaciones emergentes que en el orden mundial se presentan: los flujos migratorios, el abandono escolar, el desplazamiento como fenómeno recurrente y las dificultades de acceso a los sistemas educativos, entre otros, siguen siendo tarea de los diferentes actores que tienen que ver con el campo educativo y deben continuar proponiéndose como puntos fundamentales dentro de las agendas públicas.

En ese sentido, cuestionarse frente a estas realidades es imprescindible para entender los problemas educativos en contextos específicos y generales, al igual que identificar, desde los diversos ámbitos del conocimiento representados en los paradigmas de la investigación, las posibilidades dialécticas para acercarse al entendimiento de los fenómenos en la pluralidad contextual, y así generar y fortalecer las prácticas científicas que trasciendan los enfoques reduccionistas que han primado frente a lo educativo y posicionar nuevas apuestas que, dirigiendo las miradas hacia la interacción, a la construcción compartida de los conocimientos y a las transformaciones sociales, puedan aportar en las soluciones para mitigar o superar los problemas educativos actuales.

La investigación educativa tiene como reto pensarse para trascender los problemas emergentes en la educación, constituirse como una posibilidad para abrir caminos hacia la reflexión, la comprensión de los hechos, las causas y los diferentes fenómenos que integran lo educativo, apoyarse en creencias, valores y conocimientos (paradigmas) que posibiliten el 


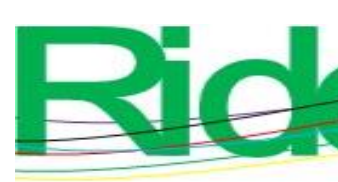

Revista Iberoamericana para la
Investigación y el Desarrollo Educativo
ISSN $2007-7467$

acercamiento a las realidades y aporten de manera significativa en la consolidación de una de las muchas miradas frente a problemáticas que coexisten en la educación.

Para los investigadores, como tercer y último punto en este artículo, continúa el reto de darle sentido a la práctica investigativa para asumir, desde una posición autocrítica, los aspectos que ameriten resignificarse o reinventarse, entendiendo esta tarea como un ejercicio consciente frente a los cambios y necesidades de las sociedades contemporáneas.

Por último, vale la pena enfatizar en la reflexión pedagógica como el eje articulador de la actividad investigativa y como el territorio fundamental para asumir el análisis de los problemas de la educación, y procurar la apertura discusiones que, partiendo del aporte de los diversos paradigmas, provean de sentido y significado los campos de estudio y direccionen la búsqueda de nuevas comprensiones y acercamientos a las realidades.

\section{Referencias}

Alvarado, L. y García, M. (2008). Características más relevantes del paradigma sociocrítico. $\mathrm{Su}$ aplicación en investigaciones de educación ambiental y de enseñanza de las ciencias realizadas en el Doctorado de Educación del Instituto Pedagógico de Caracas. Revista Universitaria de Investigación, 9(2), 187-202.

Capra, F. (1998): La trama de la vida. Una nueva perspectiva de los sistemas vivos. En: Editorial Anagrama. Barcelona- España.

Flores, M. (2004). Implicaciones de los paradigmas de investigación en la práctica educativa. Revista Digital Universitaria, 5(1), 2-9.

Freire, P. (1980). La educación como práctica de la libertad. México: Siglo XXI.

Gadamer, H. (1995). El giro hermenéutico. Madrid, España: Ediciones Cátedra.

Gómez, V. H. (2017). Ciencia y tecnología: cambios, transformaciones y retos. Revista Ciencias Estratégicas, 25(37), 9-12.

Habermas, J. (1973). Conocimiento e interés. Ideas y Valores, (42-45), 61-76.

Hoyos, G. (1986). Comunicación y mundo de la vida. Ideas y Valores, 35(71-72), 73-105. Recuperado de https://revistas.unal.edu.co/index.php/idval/article/view/18914.

Kuhn, T. (1962). La estructura de las revoluciones científicas. México: Fondo de Cultura Económica.

Lukas, J. y Santiago, K. (2009). Evaluación educativa (2. ${ }^{a}$ ed.). Madrid, España: Alianza. 


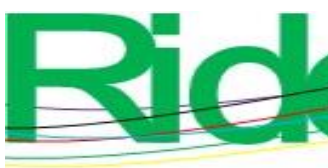

Revista Iberoamericana para la
Investigación y el Desarrollo Educativo
ISSN $2007-7467$

Marín, L. (2007). La noción de paradigma. Signo y Pensamiento, 25(50), 34-45. Recuperado de https://revistas.javeriana.edu.co/index.php/signoypensamiento/article/view/3705.

Martínez, A. y Ríos, F. (2006). Los conceptos de conocimiento, epistemología y paradigma, como base diferencial en la orientación metodológica del trabajo de grado. Cinta moebio, 25, 111-121.

Martínez, V. (2013). Paradigmas de investigación. Manual multimedia para el desarrollo de trabajos de investigación. Una visión desde la epistemología dialéctico crítica. México: Posgrado Integral en Ciencias Sociales de la Universidad de Sonora. Recuperado de https://pics.unison.mx/wpcontent/uploads/2013/10/7_Paradigmas_de_investigacion_2013.pdf.

Medina, A. y Ballano, S. (2015). Las aportaciones de Schütz, Berger y Habermas al debate sobre la objetividad de conocimiento. Andamios. Revista de investigación Social, 12(28), 241-264.

Medina, C. (2001). Paradigmas de la Investigación sobre lo cuantitativo y lo cualitativo. Ciencia e Ingeniería Neogranadina, (10), 79-84.

Meza, C. V. (2013). Relacionándonos en un mundo diverso: un problema social contemporáneo. Hallazgos, 10(19), 47-66.

Najmanovich, D. (19 de octubre de 1991). Interdisciplina y nuevos paradigmas. Página/12. Recuperado de https://www.yumpu.com/es/document/read/38246695/interdisciplina-y-nuevosparadigmas-la-ciencia-de-fin-de-siglo.

Organización de las Naciones Unidas [ONU]. (2016). Una humanidad: nuestra responsabilidad compartida. España: Organización de las Naciones Unidas.

Ricoy, C. (2006). Contribución sobre los paradigmas de investigación. Educação. Revista do Centro de Educação, 31(1), 11-22.

Vasilachis, I. (2006). Estrategias de investigación cualitativa. Barcelona, España: Gedisa. 


\begin{tabular}{|c|c|}
\hline Rol de Contribución & Autor (es) \\
\hline Conceptualización & Sorangela Miranda Beltrán y Jorge Alexander Ortiz Bernal \\
\hline Metodología & Sorangela Miranda Beltrán y Jorge Alexander Ortiz Bernal \\
\hline Software & No aplica \\
\hline Validación & $\begin{array}{l}\text { Comité de Investigaciones. Facultad de Ciencias de la } \\
\text { Educación. Universidad Externado de Colombia. }\end{array}$ \\
\hline Análisis Formal & No aplica \\
\hline Investigación & Sorangela Miranda Beltrán y Jorge Alexander Ortiz Bernal \\
\hline Recursos & Sorangela Miranda Beltrán y Jorge Alexander Ortiz Bernal \\
\hline Curación de datos & Sorangela Miranda Beltrán y Jorge Alexander Ortiz Bernal \\
\hline $\begin{array}{l}\text { Escritura - Preparación del } \\
\text { borrador original }\end{array}$ & Sorangela Miranda Beltrán y Jorge Alexander Ortiz Bernal \\
\hline $\begin{array}{l}\text { Escritura - Revisión y } \\
\text { edición }\end{array}$ & Sorangela Miranda Beltrán y Jorge Alexander Ortiz Bernal \\
\hline Visualización & Sorangela Miranda Beltrán y Jorge Alexander Ortiz Bernal \\
\hline Supervisión & $\begin{array}{l}\text { Cecilia Dimaté Rodríguez. Decana. Facultad de Ciencias de la } \\
\text { Educación. }\end{array}$ \\
\hline $\begin{array}{l}\text { Administración de } \\
\text { Proyectos }\end{array}$ & Sorangela Miranda Beltrán y Jorge Alexander Ortiz Bernal \\
\hline Adquisición de fondos & Sorangela Miranda Beltrán y Jorge Alexander Ortiz Bernal \\
\hline
\end{tabular}




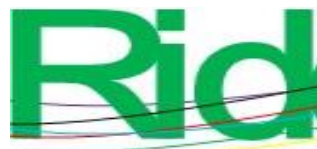

2

\begin{abstract}
.
\end{abstract}
Sorangela Miranda Beltrán

Postdoctora en Gestión y Administración de Planteles Educativos por el Centro de Estudios e Investigaciones para el Desarrollo Docente-Cenid (México), Doctora en Ciencias Sociales y Humanas por la Pontificia Universidad Javeriana (Colombia), Magíster en Administración

y Supervisión Educativa por la Universidad Externado de Colombia y Licenciada en Educación Especial por la Universidad Pedagógica Nacional (Colombia). Actualmente se desempeña como docente en la Maestría en Educación y en la Maestría en Evaluación y Aseguramiento de la Calidad de la Educación, ambas de la Universidad Externado de Colombia.

\title{
Jorge Alexander Ortiz Bernal
}

Doctor en Educación por la Universidad de la Salle (Costa Rica), Máster en Dirección de Centros Educativos por el Centro Universitario Villanueva de la Universidad Complutense de Madrid (España), Magíster en Gestión y Evaluación Educativa por la Universidad Externado de Colombia y Licenciado en Ciencias Sociales por la Universidad Antonio Nariño (Colombia). Actualmente se desempeña como docente en la Maestría en Educación y en la Maestría en Evaluación y Aseguramiento de la Calidad de la Educación, ambas de la Universidad Externado de Colombia. 